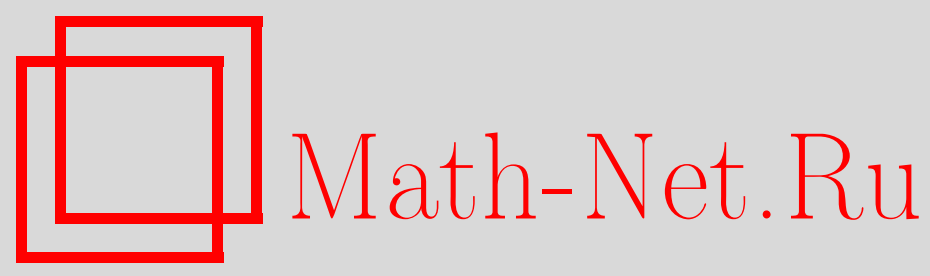

Н. Н. Андреев, Минимальный дизайн 11-го порядка на трехмерной сфере, Матем. заметки, 2000, том 67, выпуск 4, 489-497

DOI: https://doi.org/10.4213/mzm863

Использование Общероссийского математического портала Math-Net.Ru подразумевает, что вы прочитали и согласны с пользовательским соглашением http://www . mathnet.ru/rus/agreement

Параметры загрузки:

IP : 34.229 .108 .108

26 апреля 2023 г., $17: 47: 32$

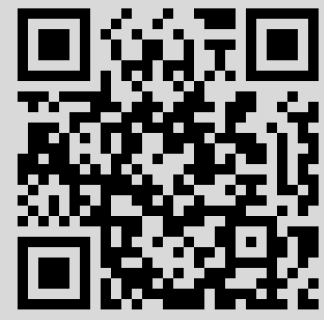




\section{МИНИМАЛЬНЫЙ ДИЗАЙН 11-ГО ПОРЯДКА НА ТРЕХМЕРНОЙ СФЕРЕ}

\section{Н. Н. Андреев}

В статье доказьвается, что 120 вершин правильного четырехмерного многогранника с символом Шлефоли $\{3,3,5\}$ являются минимальным сферическим дизайном 11-го порядка на $S^{3}$. Показывается, что заряды, расположенные в этих вершинах, доставляют минимум потенциальной энергии системы из 120 равных зарядов, расположенных на $S^{3}$.

Библиография: 23 названия.

Пусть $S^{m-1}=\left\{\left(x_{1}, x_{2}, \ldots, x_{m}\right) \in \mathbb{R}^{m} \mid x_{1}^{2}+x_{2}^{2}+\cdots+x_{m}^{2}=1\right\}$ - единичная сфера в $m$-мерном евклидовом пространстве; для $x, y \in \mathbb{R}^{m}$ через $x y$ обозначим скалярное произведение векторов; $|x|=\sqrt{x x}$.

Конечное множество точек $X=\left\{x^{(k)}\right\}_{k=1}^{N} \subset S^{m-1}$ называется взвешенным дизайном порядка q, если кубатурная формула

$$
\frac{1}{\operatorname{mes} S^{m-1}} \int_{S^{m-1}} f(x) d x=\sum_{k=1}^{N} p_{k} f\left(x^{(k)}\right), \quad \sum_{k=1}^{N} p_{k}=1,
$$

верна для всех алгебраических полиномов $f(x)$ степени не вьше $q$ (под степенью монома $x_{1}^{\alpha_{1}} \cdots x_{m}^{\alpha_{m}}$ понимается сумма показателей $\left.\alpha_{1}+\cdots+\alpha_{m}\right)$. В этой статье рассматривается лишь случай положительных весов:

$$
\forall k \quad p_{k}>0 \text {. }
$$

Основная задача состоит в нахождении множеств $X$ и весов $\left\{p_{k}\right\}$, для которых вьполняется (1). Особьй интерес представляют множества $X$, содержащие минимальное количество точек, необходимое для вьполнения (1). Такие множества назьваются минимальными взвешенными дизайнами. Отдельный интерес представляет случай равных весов. В этом случае употребляются термины дизайн и минимальный дизайн.

Простейший минимальньй дизайн - две противоположные точки сферы $S^{m-1}$, являющиеся минимальным дизайном первого порядка. Примерами дизайнов являются вершины некоторых правильных многогранников. Так правильньй симплекс, вписанный в сферу $S^{m-1}$ (множество из $m+1$ точки с равными попарными расстояниями), является минимальным дизайном 2-го порядка для любого $m$. Октаэдр, вписанньй в сферу

Работа выполнена при поддержке Российского фонда фундаментальных исследований, грант № 99-01-01210. 
(множество точек пересечения $S^{m-1}$ с координатными осями), является минимальным дизайном 3-го порядка в любой размерности. Вершины икосаэдра образуют минимальный дизайн порядка 5. В то же время вершины куба и додекаэдра, являясь дизайнами соответственно 3-го и 5-го порядка, не являются минимальными. Все вьшеперечисленные минимальные дизайны являются минимальными и в классах взвешенных дизайнов соответствующих порядков. В общей ситуации это не всегда так (см., например, [1]).

Количество точек минимального взвешенного дизайна порядка $q$ на $S^{m-1}$ будем обозначать $N(m, q)$, а минимального дизайна с равными весами $-N^{*}(m, q)$; очевидно, $N^{*}(m, q) \geqslant N(m, q)$. Оценкам снизу мощности минимальных дизайнов посвящен ряд работ. В [2] получена оценка

$$
N^{*}(m, q) \geqslant \begin{cases}\left(\begin{array}{c}
m+s-1 \\
m-1
\end{array}\right)+\left(\begin{array}{c}
m+s-2 \\
m-1
\end{array}\right), & q=2 s \\
2\left(\begin{array}{c}
m+s-1 \\
m-1
\end{array}\right), & q=2 s+1 .\end{cases}
$$

При $m=4$ и $q=11$ из нее следует неравенство $N^{*}(4,11) \geqslant 112$. В работах [3], [4] получена оценка $N^{*}(4,11) \geqslant 117$. В то же время 120 вершин правильного многогранника четырехмерного евклидова пространства с символом Шлефли $\{3,3,5\}[5]-8$ вершин вида $( \pm 1,0,0,0),(0, \pm 1,0,0),(0,0, \pm 1,0),(0,0,0, \pm 1) ; 16$ вершин вида $( \pm 1 / 2, \pm 1 / 2, \pm 1 / 2$, $\pm 1 / 2)$ и 96 вершин, полученных из 8 точек вида $( \pm(\sqrt{5}+1) / 4, \pm 1 / 2, \pm(\sqrt{5}-1) / 4,0)$ четньми перестановками координат, - являются [6] дизайном 11-го порядка и, значит, $N^{*}(4,11) \leqslant 120$. Совокупность приведенных 120 точек будет обозначаться $\mathfrak{M}$.

За последние годы доказана минимальность лишш нескольких дизайнов (см. обзор [7] и работу [1]). В этой статье доказывается минимальность еще одного дизайна в классе взвешенных дизайнов.

Теорема 1. Пусть множество $X=\left\{x^{(k)}\right\}_{k=1}^{N} \subset S^{3}$ есть минимальный взвеиенный дизайн порядка 11 с положстельными весами. Тогда

$$
N(4,11)=N^{*}(4,11)=120 \text {. }
$$

Для доказательства оценок снизу будет использоваться соображение Дельсарта [8], получившее развитие в работах [3], [9]-[13]. Оно базируется на свойствах системы многочленов Гегенбауэра $\left\{G_{\nu}^{(m)}(t)\right\}_{\nu=0}^{\infty}$, ортогональных на интервале $(-1,1)$ с весом $\left(1-t^{2}\right)^{(m-3) / 2}$ и с нормировкой $G_{\nu}^{(m)}(1)=1$ :

$$
\begin{gathered}
G_{0}^{(m)}(t)=1, \quad G_{1}^{(m)}(t)=t, \quad G_{2}^{(m)}(t)=\frac{m t^{2}-1}{m-1}, \ldots, \\
(\nu+m-2) G_{\nu+1}^{(m)}(t)=(2 \nu+m-2) t G_{\nu}^{(m)}(t)-\nu G_{\nu-1}^{(m)}(t) .
\end{gathered}
$$

В геометрических задачах используется положительная определенность многочленов Гегенбауэра $\left[14\right.$, с. 318]: для любого конечного множества точек $x^{(1)}, \ldots, x^{(N)}$ из $S^{m-1}$, любого $\nu \in \mathbb{N}$ и любых $\xi_{1}, \ldots, \xi_{N} \in \mathbb{C}$ справедливо неравенство

$$
\sum_{k, l=1}^{N} G_{\nu}^{(m)}\left(x^{(k)} x^{(l)}\right) \xi_{k} \bar{\xi}_{l} \geqslant 0 .
$$


В дальнейшем нам будет удобнее пользоваться эквивалентным определением понятия взвешенного дизайна порядка $q$ как множества точек $X=\left\{x^{(k)}\right\}_{k=1}^{N} \subset S^{m-1}$, для которого справедливы равенства

$$
\sum_{k, l=1}^{N} G_{\nu}^{(m)}\left(x^{(k)} x^{(l)}\right) p_{k} p_{l}=0, \quad \nu=1,2, \ldots, q .
$$

Наряду со взвешенными дизайнами порядка $q$, рассматриваемьми вьше, будем использовать понятие $D$-дизайна, по-видимому введенное Дельсартом (см. [15]). Множество точек $X=\left\{x^{(k)}\right\}_{k=1}^{N} \subset S^{m-1}$ называется взвешенным $D$-дизайном, где $D \subset \mathbb{N}$ есть подмножество натурального ряда, если справедливы равенства

$$
\sum_{k, l=1}^{N} G_{\nu}^{(m)}\left(x^{(k)} x^{(l)}\right) p_{k} p_{l}=0, \quad \nu \in D .
$$

При $D=\{1,2, \ldots, q\}$ это есть классический взвешенный дизайн порядка $q$. Многие дизайны порядка $q$ являются $D$-дизайнами для более широкого, чем $\{1,2, \ldots, q\}$, множества $D$, и, как будет следовать из дальнейшего, этот факт иногда оказьвается важньп при оценке мощности дизайнов и в других вопросах. Так, например, М, являясь, как

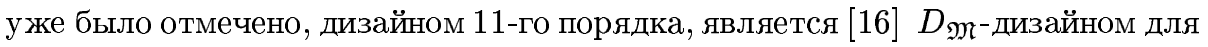

$$
\begin{aligned}
D_{\mathfrak{M}}= & \{\text { все нечетные натуральные числа }\} \\
& \cup\{2,4,6,8,10,14,16,18,22,26,28,34,38,46,58\} .
\end{aligned}
$$

Через $N(m, D)$ будем обозначать количество точек минимального взвешенного $D$-дизайна. В статье будут сформулированы экстремальные задачи теории функций, позволяющие оценивать снизу мощность $D$-дизайнов.

Конструкции, являющиеся дизайнами, оказываются решением некоторых задач дискретной геометрии об экстремальном расположении точек на сфере. Рассмотрим задачу о расположении зарядов на сфере, минимизирующем потенциальную энергию системы. Пусть в точках $\left\{x^{(k)}\right\}_{k=1}^{N} \subset S^{m-1}$ расположены единичные заряды. Функционал, обобщающий потенциальную энергию системы зарядов, расположенных в 3-мерном евклидовом пространстве, имеет вид

$$
W\left(m, N, x^{(1)}, \ldots, x^{(N)}\right)=\sum_{\substack{k, l=1 \\ k \neq l}}^{N} \frac{1}{\left|x^{(k)}-x^{(l)}\right| m-2}, \quad m \geqslant 3 .
$$

Требуется найти расположение зарядов на сфере, минимизирующее этот функционал, т.е. величину

$$
W(m, N)=\inf _{\left\{x^{(k)}\right\}_{k=1}^{N} \subset S^{m-1}} W\left(m, N, x^{(1)}, \ldots, x^{(N)}\right),
$$

и экстремальную конструкцию. История задачи начинается с экспериментов Томсона и описана в [17]-[19]. Значения параметров $m$ и $N$, при которьх решения известны, приведены в [20]. Следующая теорема дает решение еще одного частного случая этой задачи. 
Теорема 2. Пусть $m=4 u N=120$. Тогда $W(4,120)=10790 ; и$ әкстремальная конструкиия задается вершинами правильного многогранника с символом Шлефли $\{3,3,5\}$.

Перейдем к доказательству теорем.

ДоКАЗАТЕЛЬСТво ТЕОРЕмЫ 1. Вершины рассматриваемого многогранника являются дизайном 11-го порядка [6] и, следовательно, $N^{*}(4,11) \leqslant 120$.

Оценку снизу получим в общем случае - для $D$-дизайнов при произвольных $m$ и $D$. Идея получения оценки заимствована из [2], [3]. Отметим, что, хотя в [3] рассматривается только случай классических дизайнов с равными весами, все рассуждения и результаты верны и для случая взвешенных дизайнов с положительными весами.

Рассмотрим класс $\mathscr{K}(m, D)$ непрерьвных на отрезке $[-1,1]$ функций $f(t)$, удовлетворяющих условиям:

1) $f(t) \geqslant 0,-1 \leqslant t \leqslant 1, f(1)>0$;

2) $f(t)=\sum_{\nu=0}^{\infty} \widehat{f}_{\nu} G_{\nu}^{(m)}(t)$, где $\widehat{f}_{\nu} \leqslant 0$ при $\nu \in \mathbb{N} \backslash D$.

Предположим, что множество $X=\left\{x^{(k)}\right\}_{k=1}^{N} \subset S^{m-1}$ является взвешенньм $D$-дизайном с положительными весами $\left\{p_{k}\right\}_{k=1}^{N}$. Из положительности функции $f \in \mathscr{K}(m, D)$, весов $p_{k}$, а также из неравенства между средним арифметическим и средним квадратичным найдем

$I=\sum_{k, l=1}^{N} f\left(x^{(k)} x^{(l)}\right) p_{k} p_{l} \geqslant \sum_{k=1}^{N} f\left(x^{(k)} x^{(k)}\right) p_{k}^{2}=f(1) \sum_{k=1}^{N} p_{k}^{2} \geqslant f(1) \frac{\left(\sum_{k=1}^{N} p_{k}\right)^{2}}{N}=\frac{f(1)}{N}$.

С другой стороны,

$$
\begin{aligned}
I & =\sum_{k, l=1}^{N} f\left(x^{(k)} x^{(l)}\right) p_{k} p_{l}=\sum_{\nu=0}^{\infty} \widehat{f}_{\nu} \sum_{k, l=1}^{N} G_{\nu}^{(m)}\left(x^{(k)} x^{(l)}\right) p_{k} p_{l} \\
& =\widehat{f}_{0} \sum_{k=1}^{N} p_{k} p_{l}+\sum_{\nu \in D} \widehat{f}_{\nu} \sum_{k, l=1}^{N} G_{\nu}^{(m)}\left(x^{(k)} x^{(l)}\right) p_{k} p_{l}+\sum_{\nu \in \mathbb{N} \backslash D} \widehat{f}_{\nu} \sum_{k, l=1}^{N} G_{\nu}^{(m)}\left(x^{(k)} x^{(l)}\right) p_{k} p_{l}
\end{aligned}
$$

Так как $X$ - взвешенный $D$-дизайн с весами $\left\{p_{k}\right\}$, то вторая сумма равна нулю. В силу положительной определенности многочленов Гегенбауэра и неположительности коэффициентов $Ф$ урье $\widehat{f}_{\nu}$, участвующих в третьей сумме, она оказьвается неположительной и, следовательно, справедливо неравенство

$$
I \leqslant \widehat{f_{0}} \sum_{k, l=1}^{N} p_{k} p_{l}=\widehat{f}_{0}\left(\sum_{k=1}^{N} p_{k}\right)^{2}=\widehat{f_{0}}
$$

Объединяя полученные неравенства и учитьвая, что $\widehat{f}_{0}>0$ в силу условия 1$)$, приходим к оценке снизу мощности взвешенного $D$-дизайна на $S^{m-1}$ через решение экстремальной задачи теории функций

$$
N(m, D) \geqslant \sup _{f \in \mathscr{K}(m, D)} \frac{f(1)}{\widehat{f}_{0}}
$$


Для оценки снизу мощности взвешенного дизайна порядка $q$ следует рассмотреть $D=\{1,2, \ldots, q\}$. Имеем

$$
N(m, q) \geqslant \sup _{f \in \mathscr{K}(m,\{1,2, \ldots, q\})} \frac{f(1)}{\widehat{f}_{0}} .
$$

Вернемся к нашему случаю $m=4$ и $q=11$. Для доказательства того, что $\mathfrak{M}$ является минимальным дизайном 11-го порядка, будет решатся следующая экстремальная задача.

Пусть $D_{\mathfrak{M}}$ определено равенством $(2)$. Рассмотрим класс $\mathscr{K}^{*}\left(4, D_{\mathfrak{M}}\right)$ непрерывных на отрезке $[-1,1]$ функций $f(t)$, удовлетворяющих условиям:

1) $f(t) \geqslant 0,-1 \leqslant t \leqslant 1, f(1)>0$;

2) $f(t)=\sum_{\nu \in D_{\mathfrak{M}}} \widehat{f}_{\nu} G_{\nu}^{(m)}(t)$ и $\widehat{f}_{\nu} \leqslant 0$ при $\nu>12$.

Так как $\mathscr{K}^{*}\left(4, D_{\mathfrak{M}}\right) \subset \mathscr{K}(4,\{1,2, \ldots, 11\})$ ввиду рассматриваемого $D_{\mathfrak{M}}$ и условия 2$)$, то для мошности взвешенного дизайна порядка 11 на $S^{3}$ имеет место оценка

$$
N(4,11) \geqslant \sup _{f \in \mathscr{K}^{*}\left(4, D_{\mathfrak{M}}\right)} \frac{f(1)}{\widehat{f}_{0}} .
$$

Выберем функцию $f(t) \in \mathscr{K}^{*}\left(4, D_{\mathfrak{M}}\right)$ так, чтобы правая часть (5) стала максимальной. С этой целью рассмотрим семейство алгебраических многочленов

$$
\begin{aligned}
h_{\mu}(t)= & (t+1) t^{2}\left(t^{2}-\frac{1}{4}\right)^{2}\left(t^{2}-\frac{3-\sqrt{5}}{8}\right)^{2}\left(t+\frac{1+\sqrt{5}}{4}\right)^{2}\left(t-\frac{1+\sqrt{5}}{4}\right)^{2} \\
& \times\left(\frac{32768}{5}-\left(\frac{16384}{3}+\mu\right) t+\mu t^{2}\right) .
\end{aligned}
$$

Разложим их в ряд Фурье по системе многочленов Гегенбауэра

$$
h_{\mu}(t)={\widehat{\left(h_{\mu}\right)}}_{0} G_{0}^{(4)}(t)+{\widehat{\left(h_{\mu}\right)}}_{1} G_{1}^{(4)}(t)+\cdots+{\widehat{\left(h_{\mu}\right)}}_{17} G_{17}^{(4)}(t) .
$$

Имеем

$$
\begin{aligned}
& {\widehat{\left(h_{\mu}\right)_{0}}}_{0}=1 \text {, } \\
& {\widehat{\left(h_{\mu}\right)}}_{3}=\frac{118}{15}-\frac{9 \mu}{16384}, \quad{\widehat{\left(h_{\mu}\right)}}_{4}=\frac{59}{4} \text {, } \\
& {\widehat{\left(h_{\mu}\right)_{6}}}=\frac{539}{30} \text {, } \\
& {\widehat{\left(h_{\mu}\right)}}_{7}=\frac{72}{5}-\frac{\mu}{2048} \text {, } \\
& {\widehat{\left(h_{\mu}\right)}}_{9}=12 \text {, } \\
& {\widehat{\left(h_{\mu}\right)}}_{10}=\frac{77}{10} \text {, } \\
& {\widehat{\left(h_{\mu}\right)}}_{12}=0 \text {, } \\
& {\widehat{\left(h_{\mu}\right)_{13}}}_{13}=\frac{14}{5}+\frac{49 \mu}{65536} \text {, } \\
& {\widehat{\left(h_{\mu}\right)}}_{15}=\frac{8}{15}+\frac{\mu}{2048} \text {, } \\
& {\widehat{\left(h_{\mu}\right)}}_{16}=-\frac{17}{12} \text {, } \\
& \widehat{\left(h_{\mu}\right)_{2}}=\frac{149}{20} \text {, } \\
& {\widehat{\left(h_{\mu}\right)_{5}}}=\frac{64}{5}-\frac{45 \mu}{65536} \text {, } \\
& {\widehat{\left(h_{\mu}\right)_{8}}}=\frac{153}{10} \text {, } \\
& {\widehat{\left(h_{\mu}\right)}}_{11}=\frac{36}{5}+\frac{9 \mu}{16384} \text {, } \\
& {\widehat{\left(h_{\mu}\right)}}_{14}=-\frac{11}{4} \text {, } \\
& {\widehat{\left(h_{\mu}\right)}}_{17}=\frac{9 \mu}{65536} \text {. }
\end{aligned}
$$

Покажем, что многочлены $h_{\mu}(t) \in \mathscr{K}^{*}\left(4, D_{\mathfrak{M}}\right)$ при $\mu \in[-90112 / 15,-131072 / 35]$. Условие 1) будет выполнено, если квадратный трехчлен, стоящий последним множителем в определении $h_{\mu}(t)$, будет неотрищательньм на $[-1,1]$. Это имеет место при отрицательных $\mu \geqslant-90112 / 15$. При $\mu \leqslant-131072 / 35$ все коэффициенты Фурье полинома 
$h_{\mu}(t)$ с номерами большими 12 неположительны, а двеннадцатьй коэффициент и все коэффициенты с номерами большими 17 равны нулю, значит условие 2) тоже вьполнено.

Так как $h_{\mu}(1)=\sum_{\nu=0}^{17} \widehat{\left(h_{\mu}\right)}=120$ для любого $\mu \in \mathbb{R}$, любой многочлен из указанного семейства при $\mu \in[-90112 / 15,-131072 / 35]$ дает требуемую оценку $N(4,11) \geqslant 120$.

Ввиду неравенств $120 \geqslant N^{*}(4,11) \geqslant N(4,11)$ теорема 1 доказана.

Для доказательства теоремы 2 нам понадобится следующая лемма.

Лемма [21, с. 71]. Пусть $z, c, z_{1}, \ldots, z_{s} \in \mathbb{C}$, все $z_{k} \neq c$ и различны $; n_{1}, \ldots, n_{s} \in \mathbb{Z}_{+}$, $n=n_{1}+\cdots+n_{s}$. Для функиии

$$
f(z)=\frac{1}{c-z}
$$

интерполячионный полином $p_{n-1}(z)$ степени $n-1$, удовлетворяющий соотношениям

$$
p_{n-1}^{(l)}\left(z_{k}\right)=f^{(l)}\left(z_{k}\right), \quad k=1, \ldots, s, \quad l=0, \ldots, n_{k}-1,
$$

имеет вид

$$
p_{n-1}(z)=\frac{1}{c-z}-\frac{\omega(z)}{\omega(c)(c-z)}
$$

əде $\omega(z)=\left(z-z_{1}\right)^{n_{1}}\left(z-z_{2}\right)^{n_{2}} \cdots\left(z-z_{s}\right)^{n_{s}}$.

Ввиду простоты приведем доказательство леммы.

ДокАЗАТЕЛЬСТво.Приводя к общему знаменателю правую часть (7), убеждаемся, что она определяет полином по $z$ степени $n-1: \omega(z)$ есть полином по $z$ степени $n$, а в точке $z=c$ особенности нет, так как числитель тоже обрашается в нуль. Дифференцируя и подставляя $z=z_{k}$, убеждаемся, что условия (6) выполнены. В силу единственности интерполящионного полинома степени $n-1$, определенного равенствами (6), лемма доказана.

ДоКАЗАТЕЛЬСТво ТЕОРЕМЫ 2. Оценку сверху $W(4,120) \leqslant 10790$ дают заряды, расположенные в 120 вершинах многогранника $\{3,3,5\}$.

Оценим $W\left(m, N, x^{(1)}, \ldots, x^{(N)}\right)$ снизу. Пусть $\left\{x^{(1)}, \ldots, x^{(N)}\right\} \subset S^{m-1}$ - произвольный набор точек на сфере, в которых расположены единичные заряды. Так как $x^{(k)} \in$ $S^{m-1} \forall k$, то

$$
\left|x^{(k)}-x^{(l)}\right|=\sqrt{\left|x^{(k)}-x^{(l)}\right|^{2}}=\sqrt{2\left(1-x^{(k)} x^{(l)}\right)} .
$$

Тогда

$$
W\left(m, N, x^{(1)}, \ldots, x^{(N)}\right)=\sum_{\substack{k, l=1 \\ k \neq l}}^{N} \frac{1}{\left|x^{(k)}-x^{(l)}\right|^{m-2}}=\sum_{\substack{k, l=1 \\ k \neq l}}^{N} \frac{1}{\left\{2\left(1-x^{(k)} x^{(l)}\right)\right\}^{(m-2) / 2}} .
$$

Рассмотрим класс $\mathscr{F}(m)$ непрерывных на отрезке $[-1,1]$ функций $f(t)$, удовлетворяющих условиям:

1) $f(t) \leqslant 1 /\{2(1-t)\}^{(m-2) / 2},-1 \leqslant t \leqslant 1$;

2) $f(t)=\sum_{\nu=0}^{\infty} \widehat{f}_{\nu} G_{\nu}^{(m)}(t), \widehat{f}_{\nu} \geqslant 0$ для всех $\nu \in \mathbb{N}$. 
Пусть $f(t) \in \mathscr{F}(m)$. В силу условия 1$)$ можно записать

$$
\begin{aligned}
W\left(m, N, x^{(1)}, \ldots, x^{(N)}\right) & \geqslant \sum_{\substack{k, l=1 \\
k \neq l}}^{N} f\left(x^{(k)} x^{(l)}\right)=\sum_{k, l=1}^{N} f\left(x^{(k)} x^{(l)}\right)-N f(1) \\
& =\sum_{\nu=0}^{\infty} \widehat{f}_{\nu} \sum_{k, l=1}^{N} G_{\nu}^{(m)}\left(x^{(k)} x^{(l)}\right)-N f(1) .
\end{aligned}
$$

Пользуясь положительной определенностью многочленов Гегенбауэра, их нормировкой, а также неотрицательностью коэффициентов Фурье функции $f \in \mathscr{F}(m)$, продолжим оценку

$$
W\left(m, N, x^{(1)}, \ldots, x^{(N)}\right) \geqslant N^{2} \widehat{f}_{0}-N f(1) .
$$

Ввиду произвольности набора точек $\left\{x^{(1)}, \ldots, x^{(N)}\right\} \subset S^{m-1}$ приходим к оценке снизу для функционала энергии через решение экстремальной задачи

$$
W(m, N) \geqslant \sup _{f \in \mathscr{F}(m)}\left\{N^{2} \widehat{f_{0}}-N f(1)\right\} .
$$

Пусть теперь $m=4$ и $N=120$. Рассмотрим функцию

$$
y(t)=\frac{1}{2(1-t)},
$$

участвующую в определении класса $\mathscr{F}(4)$, и многочлен $h(t)$ с разложением по многочленам Гегенбауэра

$$
h(t)=\widehat{h}_{0} G_{0}^{(4)}(t)+\widehat{h}_{1} G_{1}^{(4)}(t)+\cdots+\widehat{h}_{17} G_{17}^{(4)}(t),
$$

где

$$
\begin{array}{rlll}
\widehat{h}_{0}=\frac{65869}{79200}, & \widehat{h}_{1}=\frac{132839}{99000}, & \widehat{h}_{2}=\frac{827147}{528000}, & \widehat{h}_{3}=\frac{62063}{39600} \\
\widehat{h}_{4}=\frac{49399}{35200}, & \widehat{h}_{5}=\frac{18919}{16500}, & \widehat{h}_{6}=\frac{61327}{72000}, & \widehat{h}_{7}=\frac{5647}{9900} \\
\widehat{h}_{8}=\frac{29453}{88000}, & \widehat{h}_{9}=\frac{1079}{6600}, & \widehat{h}_{10}=\frac{4363}{72000}, & \widehat{h}_{11}=\frac{289}{33000} \\
\widehat{h}_{12}=0, & \widehat{h}_{13}=\frac{161}{198000}, & \widehat{h}_{14}=\frac{97}{9600}, & \widehat{h}_{15}=\frac{203}{16500} \\
\widehat{h}_{16}=\frac{2737}{316800}, & \widehat{h}_{17}=\frac{3}{500} .
\end{array}
$$

Непосредственно могут быть проверены следующие равенства:

$$
h(t)=y(t)
$$

при

$$
\begin{aligned}
t \in & \left\{-1,-\frac{1+\sqrt{5}}{4},-\frac{1}{2}, \frac{1-\sqrt{5}}{4}, 0, \frac{-1+\sqrt{5}}{4}, \frac{1}{2}, \frac{1+\sqrt{5}}{4},\right. \\
& \left.\frac{2363-i \sqrt{169319}}{2112}, \frac{2363+i \sqrt{169319}}{2112}\right\}
\end{aligned}
$$


и

$$
h^{\prime}(t)=y^{\prime}(t)
$$

при

$$
t \in\left\{-1,-\frac{1+\sqrt{5}}{4},-\frac{1}{2}, \frac{1-\sqrt{5}}{4}, 0, \frac{-1+\sqrt{5}}{4}, \frac{1}{2}, \frac{1+\sqrt{5}}{4}\right\} .
$$

Покажем, что многочлен $h(t) \in \mathscr{F}(4)$. Условие 2$)$ выполнено в силу самого определения $h(t)$. С учетом (9) и (10) из леммы следует, что

$$
h(t)=\frac{1}{2(1-t)}-\frac{\omega(t)}{2 \omega(1)(1-t)},
$$

где

$$
\begin{aligned}
\omega(t)= & t^{2}(t+1)^{2}\left(t^{2}-\frac{1}{4}\right)^{2}\left(t^{2}-\frac{3-\sqrt{5}}{8}\right)^{2}\left(t+\frac{1+\sqrt{5}}{4}\right)^{2}\left(t-\frac{1+\sqrt{5}}{4}\right)^{2} \\
& \times\left(\left(t-\frac{2363}{2112}\right)^{2}+\frac{169319}{2112^{2}}\right) .
\end{aligned}
$$

Так как $\omega(t) \geqslant 0$ при $t \in[-1,1]$, то

$$
h(t) \leqslant \frac{1}{2(1-t)}, \quad t \in[-1,1]
$$

что и требуется в условии 1$)$ определения класса $\mathscr{F}(4)$.

Подставляя значения $\widehat{h}_{0}=65869 / 79200$ и $h(1)=1631 / 165$ в $(8)$, получаем $W(4,120) \geqslant$ 10790. Оценка снизу совпадает с оценкой сверху, что и доказывает теорему 2.

Отметим, что, как и в задаче (5), в задаче (8) экстремальным является однопараметрическое семейство многочленов, которое не приводится здесь из-за своей громоздкости.

Конфигурация точек $\mathfrak{M}$, образованная вершинами многогранника $\{3,3,5\}$, является решением ряда классических задач дискретной геометрии. В [22] (см. также [23]) показьвается, что это есть максимальньй сферический $\cos \frac{\pi}{5}$-код. Здесь было доказано, что $\mathfrak{M}$ является минимальньм сферическим дизайном 11-го порядка на $S^{3}$, а также наилучшим расположением точек в смысле задачи об энергии. Вершины рассматриваемого многогранника являются экстремальными и в некоторых других задачах дискретной геометрии, например в задаче Фейеш Тота. Так на $\mathfrak{M}$ достигаются верхние грани

$$
\sup _{\left\{x^{(k)}\right\}_{k=1}^{120} \subset S^{3}} \sum_{k, l=1}^{120}\left|x^{(k)}-x^{(l)}\right|, \quad \sup _{\left\{x^{(k)}\right\}_{k=1}^{120} \subset S^{3}} \prod_{k, l=1}^{120}\left|x^{(k)}-x^{(l)}\right| ;
$$

в частности,

$$
\begin{aligned}
\sup _{\left\{x^{(k)}\right\}_{k=1}^{120} \subset S^{3}} \prod_{k, l=1}^{120}\left|x^{(k)}-x^{(l)}\right|= & 2^{-7151} 3^{600} 5^{192}(3+\sqrt{5})^{1968}(5+2 \sqrt{5})^{336} \\
& \times(7+3 \sqrt{5})^{240}(1-\sqrt{5})^{5904} .
\end{aligned}
$$

Доказательства этих фактов по сути повторяют доказательство теоремы 2. Они требуют еще больших вычислений и поэтому здесь не приводятся.

В заключение я выражаю искреннюю благодарность В. А. Юдину, из многочисленных обсуждений с которым возникла эта статья, а также П. А. Бородину, Д. В. Горбачеву, В. И. Иванову и В.И. Левенштейну за полезные замечания. 


\section{СПИСОК ЦИТИРОВАННОЙ ЛИТЕРАТУРЫ}

[1] Hardin R. H., Sloane N. J. A. Expressing $\left(a^{2}+b^{2}+c^{2}+d^{2}\right)^{3}$ as a sum of 23 sixth powers // J. Combin. Theory. Ser. A. 1994. V. 68. P. 481-485.

[2] Delsarte P., Goethals J. M., Seidel J. J. Spherical codes and designs // Geom. Dedicata. 1977. V. 6. P. 363-388.

[3] Юдин В.А. Нижние оценки для сферических дизайнов // Изв. РАН. Сер. матем. 1997. T. 61. № 3. C. 213-223.

[4] Nikova S., Boyvalenkov P. Improvements of the lower bounds of the size of some spherical designs // Mathematika Balkanica. (to appear).

[5] Берже М. Геометрия. Т. 1. М.: Мир, 1984.

[6] Салихов Г.Н. Кубатурные формулы для гиперсферы, инвариантные относительно группы правильного 600-гранника // Докл. АН СССР. 1975. Т. 223. № 5. С. 1075-1078.

[7] Seidel J. J. Isometric embeddings and geometric designs // Discrete Math. 1994. V. 136. P. 281-293.

[8] Delsarte Ph. Bounds for unrestricted codes, by linear programing // Philips Res. Rep. 1972. V. 2. P. 272-289.

[9] Кабатянский Г. А., Л евенштейн В. И. О границах для упаковок на сфере и в пространстве // Пробл. передачи информации. 1978. Т. 14. №1. С. 3-25.

[10] Левенштейн В.И. О границах для упаковок в $n$-мерном евклидовом пространстве // Докл. AH CCCP. 1979. T. 245. C. 1299-1303.

[11] Сидельников В. М. Об экстремальных многочленах, используемых при оценках мощности кода // Пробл. передачи информации. 1980. Т. 16. № 3. С. 17-30.

[12] Юдин В.А. Минимум потенциальной энергии точечной системы зарядов // Дискретная матем. 1992. Т. 4. № 2. С. 115-121.

[13] Арестов В.В., Бабенко А.Г. О схеме Дельсарта оценки контактных чисел // Тр. МИАН. 1997. Т. 219. С. 44-73.

[14] Конвей Дж., Слоэн Н. Упаковки шаров, решетки и группы. Т. 1. М.: Мир, 1990.

[15] Delsarte Ph., Levenstein V. I. Association schemes and coding theory // IEEE Trans. Inform. Theory. 1998. V. 44. №6. P. 2477-2504.

[16] Andreev N. N., Yudin V.A. Approximation problems in discrete geometry // Advances in Multivariate Approximations / ed. W. Haussmann, K. Jetter, M. Reimer (to appear).

[17] Тот Л.Ф. Расположения на плоскости, на сфере и в пространстве. М.: Физматлит, 1958.

[18] Whyte L. L. Unique arrangements of points on a sphere // Amer. Math. Monthly. 1952. V. 59. № 9. P. 606-611.

[19] Björk G. Distributions of positive mass, which maximise a certain generalised energy integral // Ark. Math. 1956. V. 3. P. 255-269.

[20] Андреев Н. Н. Расположение точек на сфере с минимальной энергией // Тр. МИАН. 1997. T. 219. C. $27-31$.

[21] Уолш Дж. Интерполяция и аппроксимация рациональными функциями в комплексной области. М.: ИЛ, 1961.

[22] Андреев Н.Н. Один сферический код // УМН. 1999. Т. 54. № 1. С. 255-256.

[23] Ericson T., Zinoviev V. Spherical codes (to appear).

Московский государственный университет им. М.В. Ломоносова

Поступило

E-mail: andreev@mech.math.msu.su

05.04.1999

Исправленный вариант

08.07.1999 